\title{
As relações entre despesas públicas e indicadores de saúde: uma análise de correlação canônica para os estados brasileiros
}

\author{
Relations between public expenditure and health indicators: a canonic \\ correlation analysis for Brazilian states
}

\author{
Darlan Christiano Kroth e Luiz Carlos Ribeiro Neduziak*
}

\begin{abstract}
Resumo: O presente trabalho mensurou a relação entre um conjunto de indicadores de despesas públicas, pertencentes ao grupo dos determinantes sociais da saúde, e os indicadores de saúde, mediante o emprego da técnica multivariada de análise de correlação canônica. O objetivo do estudo foi avaliar os efeitos dessas despesas sobre as condições de saúde dos estados brasileiros e do Distrito Federal, na perspectiva de aprimorar as políticas públicas de saúde, contemplando a articulação de ações de diferentes áreas. Considerando que a saúde é um conceito multidisciplinar, tais indicadores sintéticos possibilitam uma análise mais ampla da condição de saúde dos estados, uma vez que capturam conjuntamente características latentes que, isoladamente, não possibilitariam o mesmo resultado. A correlação canônica, entre o primeiro par de variáveis canônicas, apresentou-se bastante elevada (95,31\%) e significativa, indicando que melhores resultados na área da saúde, bem como maior eficiência dos gastos e ganhos de economias de escala e de escopo, podem ser alcançados mediante o desenho de políticas públicas articuladas de diferentes áreas.
\end{abstract}

Palavras-chave: Economia da Saúde; Indicadores Sintéticos; Correlação Canônica; Saúde e Desenvolvimento

Abstract: This study aims to verify the relationship between public expenditures indexes, which belong to health social determinants, and health indexes, by means of multivariate analysis of canonical correlation. The objective is to evaluate the public expenditure effects on health conditions of the Brazilian states and the Federal District, in order to improve health public policy in various areas. Considering that health is a multidisciplinary concept, such synthetic indexes may help capture joint characteristics, which, individually, would not have the same outcome. The first canonical correlation showed a very high output (95.31\%), suggesting that better results in public health, as well as more efficiency in expenditures and earnings, can be reached by devising public policies in different areas.

Keywords: Health Economics; Synthetic Index; Canonical Correlation; Health and Economic Development

JEL Code: H51; C43; I15

\footnotetext{
* Respectivamente, professor da Universidade Federal da Fronteira Sul, campus Chapecó (SC). E-mail: dckroth@uffs.edu.br. Orcid: https://orcid.org/0000-0003-2664-857X; e professor da Faculdade de Educação Superior do Paraná e da PUCPR. E-mail luiz.neduziak@pucpr.br. Orcid: https://orcid.org/00000001-6978-4163.
} 


\section{Introdução}

O aumento das despesas públicas com saúde vem despertando a preocupação mundial nos últimos anos, considerando a restrição orçamentária contemporânea dos governos (efeitos da crise-2009) e a sustentabilidade desses gastos no tempo (GERDTHAM; JONSSON, 2000; OCDE, 2010). Nos países membros da OCDE, a participação média das despesas em saúde sobre o PIB atingiu 9,3\% em 2011, 3\% acima do verificado nas décadas de 1980 e 1990, no Brasil esse indicador é de $8,9 \%$. O crescimento anual médio das despesas em saúde nos últimos 15 anos vem superando o crescimento do PIB (3,9\% contra 2,6\%, respectivamente), sendo que cerca de $70 \%$ refere-se a financiamento público (OCDE, 2013). No caso brasileiro, apenas 42\% do total das despesas com saúde advém de recursos governamentais ${ }^{1}$.

Segundo $\mathrm{Xu}$ et al. (2011), os principais fatores que contribuíram para esse aumento, nos últimos anos, foram a renda, a estrutura etária da população e o efeito-Baumol (ou custo-doença) da tecnologia em saúde. A maior preocupação, porém, está no grande potencial de crescimento desses gastos no futuro, tendo em vista a transição demográfica (envelhecimento da população e maior expectativa de vida), a transição epidemiológica, o aumento dos preços da tecnologia médica e as expectativas da população ${ }^{2}$ (OCDE, 2010; XU et al., 2011).

Dadas as limitações orçamentárias, uma das maneiras de tentar equilibrar a manutenção (e ampliação) da oferta de serviços de saúde e a adequação das despesas é a eficiência dos gastos. Para a OCDE (2010), os sistemas de saúde são considerados economicamente eficientes (ou viáveis e sustentáveis) quando os benefícios dos gastos em saúde excedem seus custos.

Para Vega e Irwin (2004) e Who (2008), uma das maneiras mais viáveis/adequadas de alcançar essa eficiência no financiamento à saúde é ampliar o escopo da política de saúde, ou seja, considerando que a produção de saúde não depende apenas dos gastos em assistência à saúde, mas de um conjunto diversificado de insumos, a política de saúde deveria contemplar aspectos de

\footnotetext{
${ }^{1}$ Os gastos federais com saúde, no Brasil, quase dobraram no período de 2002 a 2014, passando de R\$ 49 bilhões para R\$ 92,3 bilhões, sendo o segundo maior item do gasto social, atrás apenas da Previdência (DATASUS, 2015).

${ }^{2}$ Os serviços de saúde pública no Brasil foram apontados recentemente como a maior preocupação dos eleitores brasileiros, de acordo com DATAFOLHA (2014), os quais consideram que os serviços de saúde prestados, no Brasil, são oferecidos de forma ineficiente.
} 
política econômica e social, abrangendo os determinantes sociais da saúde (WILKINSON; MARMOTT, 2003). A expansão da esfera da política pública em saúde pode contribuir para gerar ganhos de escala na produção de saúde, reduzindo, portanto, os custos totais na área de saúde e melhorando a eficiência dos gastos em saúde.

Considerando esse cenário, o presente trabalho busca avaliar em que medida os diferentes tipos de despesas públicas, alinhados com os determinantes sociais da saúde, tais como despesas com saúde, educação, assistência social, saneamento, gestão ambiental e esporte/lazer, relacionam-se com os indicadores de saúde disponibilizados pelo DATASUS (2015). O estudo teve como base os 26 estados da Federação e o Distrito Federal, para o ano de 2010.

Para desenvolver o objetivo proposto, foi empregada a metodologia de análise multivariada, considerando os recursos da Análise de Componentes Principais (ACP), Análise Fatorial (AF) e a Análise de Correlação Canônica (ACC). O emprego dessa metodologia pode ser considerado uma das contribuições de nosso trabalho, tendo em vista que a técnica é pouco explorada na área de avaliação de políticas públicas. Uma segunda contribuição do trabalho é a construção de seis indicadores sintéticos de saúde. Nesses termos, considerando que a saúde é um conceito multidisciplinar, tais indicadores sintéticos possibilitam uma análise mais ampla da condição de saúde dos estados, uma vez que capturam conjuntamente características latentes que, isoladamente, não possibilitariam o mesmo resultado.

O exercício empírico foi realizado em duas etapas, sendo a primeira a construção de seis indicadores sintéticos de saúde (indicadores de mortalidade, morbidade, demográfico, socioeconômico, cobertura e de recursos) através da AF. $\mathrm{Na}$ segunda etapa, foi realizada a ACC, considerando os seis indicadores sintéticos da saúde e seis indicadores de despesas públicas. Os principais resultados apontam para uma correlação elevada e significativa entre os indicadores das despesas e os indicadores sintéticos de saúde, demonstrando a viabilidade da integração de políticas de diferentes esferas/áreas para atingir melhorias na área da saúde.

O trabalho está divido em três seções, além desta introdução e da conclusão. A seção 1 realiza uma revisão de literatura, destacando a produção de saúde, seu impacto sobre a economia e o papel do financiamento público em saúde. Uma parte dessa seção é reservada para discutir o financiamento da saúde no Brasil. A seção 
2 apresenta a metodologia empregada, e a seção 3 é dedicada à discussão dos resultados da pesquisa. Por fim, têm-se as conclusões do trabalho.

\section{A produção de saúde, seus impactos sobre a economia e o papel do financiamento público de políticas pró-saúde}

A literatura de desenvolvimento econômico vem dando crescente atenção ao fator "saúde" como um dos elementos promotores/determinantes do aumento da produtividade dos indivíduos (WEIL, 2014; BHARGAVA et al., 2001; BLOOM et al., 2004, BLOOM; CANNING, 2005). Os principais canais pelos quais a saúde exerce sua contribuição são por meio de um maior vigor físico e mental dos trabalhadores, ampliando sua produtividade, bem como pela ampliação de dias saudáveis (ou dias de não-doença), que ampliam o tempo dedicado às atividades produtivas. Para Bloom et al. (2004), em países em desenvolvimento, em que a produção é trabalho-intensiva, essa situação é ainda mais crítica do que em países industrializados.

Outro canal importante é que a saúde reforça o impacto do capital humano na economia, na medida em que um melhor estado de saúde proporciona maiores condições de desenvolver habilidades e conhecimento pelos indivíduos. Por fim, salienta-se que a ampliação da expectativa de vida possibilita acentuar os ganhos advindos da experiência e aprendizado nas funções inovativas. Nesses termos, saúde e capital humano são complementares (ARORA, 2001; DIAS; RUSSO, 2014) ${ }^{3}$.

A avaliação de como a saúde desempenha seu papel sobre a atividade econômica pode ser visualizada através de uma função de produção agregada (padrão AK), conforme exposto por Bloom et al. (2004):

$$
Y=A K^{\alpha}(L v)^{\beta}
$$

Na equação 1, a produção (Y) advém da produtividade total dos fatores (A) e da quantidade dos fatores, capital (K) e mão de obra (L). Nessa função, a mão de

\footnotetext{
${ }^{3}$ Conforme Grossman (2000), o capital humano e saúde se complementam, ou se autorreforçam, na medida em que se pessoas mais saudáveis são mais produtivas, e, portanto, possuem maiores retornos, é provável que invistam mais em capital humano. Por outro lado, pessoas mais instruídas são mais hábeis na produção de saúde.
} 
obra está relacionada com um fator produtividade específico (v), que depende do log dos salários.

$$
v=e^{\phi_{1} s+\phi_{2} \exp +\phi_{3} \exp { }^{2}+\phi_{4} h}
$$

O fator produtividade $\mathrm{v}$, conforme a equação 2 , segue o padrão da equação de retornos salariais proposta por Mincer (1974), na qual o retorno depende da função exponencial dos anos de escolaridade (s), da experiência (exp) e da experiência ao quadrado $\left(\exp ^{2}\right)$. O formato da equação 2 ainda inclui o fator saúde (h) como influente nos retornos.

Nos estudos macroeconômicos, o indicador de saúde amplamente utilizado é a expectativa de vida da população. Os principais resultados apontam para uma relação positiva e significativa da saúde para a produtividade do trabalho. Por exemplo, o estudo de Bloom et al. (2004) evidenciou que o aumento em um ano na expectativa de vida promove um aumento de $4 \%$ no crescimento econômico (via aumento da produtividade do trabalho). Em Weil (2014), para cada 1\% de aumento na taxa de sobrevida de adultos, a produtividade do trabalho é ampliada em 1,68\%.

Associado ao impacto agregado da saúde na sociedade, há um conjunto de estudos que se debruça sobre o termo "h" da equação 2, que se referem aos estudos microeconômicos da produção de saúde. Esses estudos procuram avaliar em que medida a saúde é desenvolvida pelas famílias e/ou indivíduos. Nesse escopo, o elemento saúde pode estar relacionado com uma boa capacidade física e mental e/ou com a plena capacidade para realização de atividades pelos indivíduos, ou ainda, uma maior disponibilidade de dias saudáveis.

Dessa maneira, além da saúde propiciar maior bem-estar, ela também proporciona maior tempo disponível para o trabalho, resultando em maior remuneração ao indivíduo. Avaliando que a saúde proporciona retorno aos indivíduos, a saúde pode ser analisada em termos de um estoque (capital saúde, por exemplo), assim como o capital humano é tratado pela literatura. Nesse contexto, a saúde pode ser obtida através da realização de investimentos pelos indivíduos.

Um trabalho seminal nessa área, e que gerou uma considerável compreensão dos determinantes da saúde e da alocação de recursos (tempo e dinheiro) nas atividades de geração de saúde, é o de Grossman (1972). O modelo 
de produção de saúde de Grossman baseia-se na teoria de capital humano para explicar a demanda por capital saúde (H), ou como os indivíduos alocam seus recursos para produzirem saúde. Na visão de Grossman, o indivíduo não é apenas passivo (consumidor de saúde), mas também produz saúde, dedicando parcela de seu tempo e de sua renda para adquirir insumos de saúde. A saúde pode ser analisada como um bem de capital, pois não se deprecia instantaneamente.

Nesse sentido, as pessoas investem em saúde para propiciar mais dias saudáveis disponíveis para trabalhar, bem como para adquirir maior vigor físico e mental e serem mais produtivas. Nessa perspectiva, a utilidade do indivíduo seria uma função do seu estoque de saúde, ou capital saúde (H), e do consumo de outros bens $(\mathrm{X})$, conforme demonstrado pela equação 3.

$$
\mathrm{U}=\mathrm{f}(\mathrm{H}, \mathrm{X})
$$

No modelo de Grossman, o estoque de saúde $(\mathrm{H})$ pode ser avaliado como dias saudáveis de um indivíduo ou dias sem nenhuma doença. Nesse sentido, o tempo, considerado como os dias de um ano (365 dias), é o maior recurso do indivíduo. O tempo pode ser dividido em:

$$
\begin{gathered}
\mathrm{T}(365 \text { dias })=\mathrm{T}_{\mathrm{L}}+\mathrm{T}_{\mathrm{W}}+\mathrm{T}_{\mathrm{d}} \\
\mathrm{T}_{\mathrm{L}}=\mathrm{T}_{\mathrm{H}}+\mathrm{T}_{\mathrm{X}}
\end{gathered}
$$

em que: $\mathrm{T}_{\mathrm{L}}=$ número de dias dedicados ao lazer; $\mathrm{T}_{\mathrm{W}}=$ número de dias dedicados ao trabalho; $\mathrm{T}_{\mathrm{d}}=$ número de dias em que está doente/incapacitado; $\mathrm{T}_{\mathrm{H}}=$ tempo dedicado para a produção de saúde ou capital saúde $(\mathrm{H}) ; \mathrm{T}_{\mathrm{X}}=$ tempo dedicado para produção de bens domésticos.

Nesse caso, quanto menor $T_{d}$, maior será o tempo para trabalho $\left(T_{W}\right)$ ou lazer $\left(T_{L}\right)$. É através de $T_{W}$ que o indivíduo tem acesso à renda $(\mathrm{Y})$, a qual utiliza basicamente para adquirir insumos de saúde $\left(\mathrm{B}_{\mathrm{H}}\right)$ e outros bens adquiridos no mercado $\left(\mathrm{B}_{\mathrm{X}}\right)$. A restrição orçamentária torna-se:

$$
\mathrm{I}=\mathrm{p}_{\mathrm{H}} \mathrm{B}_{\mathrm{H}}+\mathrm{p}_{\mathrm{X}} \mathrm{B}_{\mathrm{X}}
$$

A acumulação de $H(\Delta H)$ depende da sua produção, que, por sua vez, é gerada pelo tempo dedicado/gasto em melhorar a saúde $\left(\mathrm{T}_{\mathrm{H}}\right)$ e pelos insumos 
adquiridos para produzir saúde $\left(\mathrm{B}_{\mathrm{H}}\right)$, tais como: dieta balanceada, esporte/academia, educação e assistência à saúde.

$$
\mathrm{H}=\varphi\left(\mathrm{T}_{\mathrm{H}}, \mathrm{B}_{\mathrm{H}}\right)
$$

Os bens domésticos (X), como, por exemplo, ficar em casa, cuidar das crianças, ir ao cinema, cozinhar, entre outras atividades, são produzidos com tempo $\left(\mathrm{T}_{\mathrm{X}}\right)$ e com insumos adquiridos no mercado $\left(\mathrm{B}_{\mathrm{X}}\right)$ : comida, roupas, automóvel.

$$
\mathrm{X}=\delta\left(\mathrm{T}_{\mathrm{X}}, \mathrm{B}_{\mathrm{X}}\right)
$$

Nesse caso, o indivíduo está produzindo dois tipos de bens (saúde e doméstico) e necessita de tempo e insumos para produzir tais bens. Assim, quanto mais tempo o indivíduo dedica para produzir saúde, mais dias saudáveis ele terá e, portanto, poderá ampliar sua renda dedicando mais tempo ao trabalho, ou, caso contrário, poderá desfrutar de mais lazer. Dessa maneira, o problema do indivíduo seria maximizar a equação 3 sujeito às restrições dadas pelas equações 4 e 6 .

Para Grossman (2000), há uma forte inter-relação entre saúde e educação. A educação pode melhorar a eficiência com que uma pessoa produz saúde. A educação tornaria os investimentos em saúde mais produtivos, necessitando de menos tempo para produzir saúde.

Considerando o modelo de Grossman, verifica-se um papel destacado para a política pública na promoção de saúde, através da oferta ou subsídio do elemento $\mathrm{B}_{\mathrm{H}}$ (insumos de saúde), possibilitando assim maiores incentivos para os indivíduos produzirem saúde ou maior facilidade (barateamento dos insumos de saúde) para a produção de saúde. O fornecimento ou subsídio público de insumos à saúde é justificado pelos resultados que a saúde proporciona, como, por exemplo, o elevado retorno social (em termos de produtividade), a ampliação do bem-estar da sociedade e os seus efeitos multiplicadores sobre a atividade econômica (geração de emprego e renda), tendo em vista a grande participação desse setor na economia (OCDE, 2010).

De acordo com Arora (2001), deve-se ressaltar que a saúde é um produto multifacetado, dependendo de vários tipos de insumos para sua produção (equação 7). Nesse sentido, Wilkinson e Marmott (2003) apresentam o gradiente social, isto 
é, os determinantes sociais da saúde, que vão além da assistência médica, no qual podem ser incluídos a renda, a educação, o suporte às crianças, condições de moradia e de trabalho, infraestrutura pública (saneamento e mobilidade urbana) e os hábitos de vida (dieta equilibrada, exercícios e ausências de vícios). Os determinantes sociais dão maior ênfase a aspectos socioeconômicos, pois, segundo essa literatura, são as condições sociais e econômicas que fazem as pessoas ficarem doentes e, portanto, necessitarem de cuidados médicos.

Nesse contexto, Vega e Irwin (2004) e Kickbusch (2007) advogam que as políticas para a área de saúde devem contemplar aspectos de política econômica e social, ou seja, as políticas públicas podem agir para melhorar a saúde através de mudanças no ambiente social. Corroborando com essa visão, Mackenbach et al. (2007) apontam que na vida há várias fases importantes (infância, adolescência, trabalho e envelhecimento), e pessoas desfavorecidas em cada uma dessas fases estarão em maior risco na fase seguinte, sobrecarregando e onerando o sistema de saúde. Nesse sentido, as políticas públicas (de saúde) devem assegurar uma rede de segurança e garantir trampolins para compensar desvantagens em estágios anteriores.

Dessa maneira, políticas públicas que consideram uma gama maior de insumos pró-saúde, para além da assistência à saúde, podem contribuir para gerar ganhos de escala na produção de saúde (reduzindo, portanto, os custos na área de saúde) e maior eficiência dos gastos em saúde.

\subsection{O financiamento da saúde no Brasil}

\subsubsection{A criação do Sistema Único de Saúde e seu modelo de financiamento}

O Sistema Único de Saúde (SUS) no Brasil foi estabelecido pela Constituição Federal (CF) de 1988 (Arts. 196 a 200) e regulamentado pelas leis n. 8.080/1990 e 8.142/1990. Concebido para ser um sistema de saúde universal, integral, descentralizado, regionalizado e com controle social (constituindo-se nos cinco pilares do SUS), o SUS inova também por contemplar um conceito de saúde mais amplo, abrangendo os determinantes sociais da saúde e por fazer parte de uma política de inclusão social (OCKÉ-REIS, 2009; MELAMED, 2011). Para atender esses objetivos, o modelo de financiamento do SUS passa a ser o modelo universalista unificado (ou modelo anglo-saxão), no qual a saúde é financiada por impostos gerais. 
Mais especificamente, a CF 1988 definiu que o SUS seria financiado pelo Orçamento da Seguridade Social (OSS) que contempla, além da saúde, a previdência e a assistência social, que possui como fonte de recursos as contribuições sociais. O SUS também seria financiado com recursos da União, estados e municípios (Art. 198 da CF 1988), que mais tarde seria regulamentado pela Lei n. 141/2012.

A lei n. 141/2012 é resultado de uma intensa disputa política pela vinculação mínima de recursos ao SUS, materializada na discussão da Emenda Constitucional (EC) n. 29/2000. Para Servo et al. (2011), a busca pela vinculação se deu ainda em âmbito da CF 1988 na qual pretendia garantir o mínimo de 30\% do OSS. Em virtude da preferência dada aos compromissos assumidos pela Previdência, o OSS não foi suficiente para financiar a saúde, a qual entra em crise em 1993 (quando foi socorrida com recursos do FAT). É nesse momento que se abriu a discussão pela criação de fontes estáveis de recursos, resultando na criação da CPMF em $1996^{4}$.

Em 2000, através da criação da EC 29, ficaram estabelecidos percentuais mínimos de aplicação por parte dos entes federados $(12 \%$ da arrecadação de impostos e receitas do FPE, excluídas as parcelas de transferência a municípios para os estados e o Distrito Federal; $15 \%$ da arrecadação de impostos e receitas com FPM e fundos estaduais para os municípios; e, para a União, o valor apurado na área da saúde no ano anterior acrescido da variação nominal do PIB) que garantiram uma aplicação estável e crescente de recursos para a saúde. O volume de recursos, porém, sofreu algumas perdas, em virtude da utilização de vários subterfúgios pelos entes federados para a não aplicação de montante exigido. ${ }^{5}$

Através da lei n. 141/2012, os percentuais previstos pela EC 29 foram ratificados bem como ficou definido o que seriam as Ações e Serviços Públicos de Saúde (ASPS), nos quais deveriam ser aplicados os recursos governamentais da área da saúde. Também foram estabelecidas regras de fiscalização e controle do

\footnotetext{
${ }^{4}$ Criada com o objetivo de ampliar os recursos para a área da saúde, a CPMF representou, em média, 30\% do total do orçamento federal destinado à saúde, enquanto esteve em vigor (1996 a 2007). Nesse período, a CPMF não se configurou como ampliação de recursos, mas apenas como manutenção do montante alocado ao longo dos anos para a saúde (PIOLA, et. al., 2013).

${ }^{5}$ No caso da União, houve questionamento sobre a base de cálculo para apurar os valores de cada ano, que ficou conhecido como o debate sobre a base fixa versus base móvel (CONASS, 2011). Nos estados e municípios, a indefinição do que seria ASPS, apesar de normatizado pela Resolução n. 322/2003, resultou que recursos de outras áreas, como coleta de lixo e saneamento, fossem contabilizados como sendo da saúde (CONASS, 2006).
} 
uso desses recursos. Em que pese essa nova legislação, é consensual por parte da literatura de financiamento da saúde (MEDICI, 1995; SERVO et al., 2011; PIOLA et al., 2013) que o SUS ainda não possui recursos suficientes para garantir um modelo universal, integral e equânime, resultando em grave ineficiência da prestação de serviços de saúde, excluindo assim boa parte da população ${ }^{6}$.

\subsubsection{O desempenho do financiamento do SUS no período recente}

De acordo com a literatura de financiamento da saúde, pode-se apontar cinco fatos estilizados do financiamento da saúde no Brasil: i) quantidade de recursos insuficiente para manter um sistema universal, integral e gratuito; ii) baixa participação de recursos da União; iii) falta de clareza nos critérios de rateio dos recursos entre os entes e a falta de inequidade na distribuição; iv) falhas na regulação do setor privado; e v) má gestão dos recursos.

Poder-se-ia incluir ainda a instabilidade nas fontes de recursos para o SUS (luta pela vinculação de recursos) e a falta de regulação sobre o que são e o que não são ASPS, porém a Lei n. 141/2012 resolve em grande medida essas questões.

Com relação à insuficiência de recursos, pode-se apontar que o país gasta cerca de $8,9 \%$ do PIB na área da saúde, sendo que apenas $42 \%$ são oriundos do setor público. Países com sistemas de saúde parecidos com o do Brasil, como Canadá, Reino Unido e Austrália, gastam 11,2\%, 9,4\% e 8,9\% do PIB, respectivamente, sendo que cerca de $70 \%$ desses recursos são de origem pública. Os valores per capita, no Brasil, também são pequenos, comparados com outros países, mesmo em relação a países em desenvolvimento - o Brasil gasta em termos per capita US\$ 837, Canadá US\$ 3.900 e a Argentina US\$ 1.322, (sendo 51\% públicos) dólares em PPP (OCDE, 2013). Em termos de participação dos gastos sociais totais, a saúde participava com $14 \%$ em 2004, passando para $11,7 \%$ em 2014 (MPOG, 2014).

De qualquer maneira, os recursos para a área da saúde cresceram significativamente a partir do ano 2000 através da EC 29, ampliando em 134\% em termos reais, de 2000 a 2011, conforme Piola et al. (2013). Essa variação positiva

\footnotetext{
${ }^{6}$ Segundo dados do CONASS (2011), apenas $28,6 \%$ da população usa exclusivamente o SUS e $61,5 \%$ utiliza o SUS e o segmento privado. Essa última forma de utilização ocorre porque a maioria dos planos de saúde privados não cobre o atendimento e tratamento de doenças crônicas e/ou de alta complexidade. Dessa maneira, o SUS acaba oferecendo tais serviços e exames mais complexos.
} 
foi possível devido ao maior incremento de despesas pelos estados (+223\%) e municípios (+220\%), em contrapartida da União (+75\%). Nesses termos, ampliouse a participação dos entes em comparação com a União; por exemplo, a participação dos municípios nas despesas totais em saúde passou de $21,7 \% \mathrm{em}$ 2000 para $29,6 \%$ em 2011, os estados passaram de $18,5 \%$ para $25,7 \%$, e o governo federal passou de $59,8 \%$ para $44,7 \%$, no mesmo período.

Tabela 1 - Despesas com Saúde pelos Estados - 2004 a 2013 - em R\$ bilhões

\begin{tabular}{|c|c|c|c|c|c|c|c|c|}
\hline Ano & $\begin{array}{c}\text { Despesas } \\
\text { totais em } \\
\text { Saúde }\end{array}$ & $\begin{array}{c}\text { Atenção } \\
\text { Básica }\end{array}$ & $\begin{array}{c}\text { Assist. } \\
\text { Hospitalar e } \\
\text { Ambulat. }\end{array}$ & $\begin{array}{c}\text { Suporte } \\
\text { Profilático e } \\
\text { Terapêutico }\end{array}$ & $\begin{array}{c}\text { Vigilância } \\
\text { Sanitária }\end{array}$ & $\begin{array}{c}\text { Vigilância } \\
\text { Epidemiol. }\end{array}$ & $\begin{array}{c}\text { Alimentação } \\
\text { e Nutrição }\end{array}$ & $\begin{array}{c}\text { Demais } \\
\text { Subfunções - } \\
\text { Saúde }\end{array}$ \\
\hline 2004 & 41.47 & 3.26 & 23.20 & 1.86 & 0.23 & 0.36 & 0.75 & 11.81 \\
\hline 2005 & 45.03 & 3.12 & 25.53 & 1.92 & 0.25 & 0.36 & 0.66 & 13.19 \\
\hline 2006 & 49.90 & 2.67 & 29.45 & 2.62 & 0.22 & 0.37 & 0.60 & 13.97 \\
\hline 2007 & 53.06 & 3.86 & 28.64 & 3.39 & 0.25 & 0.37 & 0.58 & 15.97 \\
\hline 2008 & 59.30 & 3.88 & 34.61 & 2.95 & 0.21 & 0.48 & 0.75 & 16.41 \\
\hline 2009 & 63.98 & 2.98 & 37.88 & 3.06 & 0.15 & 0.57 & 0.56 & 18.78 \\
\hline 2010 & 66.50 & 4.49 & 39.52 & 2.99 & 0.23 & 0.60 & 0.48 & 18.19 \\
\hline 2011 & 68.57 & 4.23 & 41.09 & 3.10 & 0.11 & 0.48 & 0.28 & 19.27 \\
\hline 2012 & 72.60 & 2.17 & 44.70 & 4.79 & 0.09 & 0.47 & 0.59 & 19.79 \\
\hline 2013 & 74.87 & 2.17 & 44.21 & 4.50 & 3.63 & 0.51 & 0.26 & 18.20 \\
\hline
\end{tabular}

Fonte: Tesouro Nacional (2015). Dados deflacionados pelo IPCA para o ano de 2013.

A Tabela 1 apresenta as despesas na área da saúde realizadas pelos estados, no período de 2004 a 2013. Verifica-se que a maior despesa se concentra na área de MAC, com 59\% do total, apresentando crescimento de 90,5\%, no período. As despesas com MAC eram esperadas em virtude da distribuição de atribuições dentro da estrutura do SUS. A segunda despesa com maior participação refere-se a "demais subfunções da saúde", com 24,3\% em 2014 e crescimento de 54,1\%. Esse tipo de despesa pode estar representando alguns dos itens do artigo $3^{\circ}$ da Lei n. 141/2012, que versa sobre as ASPS, que inclui, por exemplo, despesa com pessoal, capacitação, gestão do sistema e ações de apoio administrativo. Outras despesas que tiveram incremento significativo referem-se a vigilância sanitária $(+1.476 \%)$ e suporte profilático e terapêutico $(+141,1 \%)$.

De acordo com Marques e Mendes (2005) e Piola et al. (2013), a baixa participação da União é questionada pelos seguintes aspectos: aumento da arrecadação, manutenção da participação relativa dos gastos em saúde como percentual do PIB, ampliação dos gastos sociais e redução da participação da saúde 
nos gastos sociais. Apesar dessa situação, verificou-se um incremento de $75 \%$ na área da saúde de 2000 a 2011 (ou, 56\% se considerar o período de 2004 a 2014), o que coloca a saúde atualmente como o segundo maior gasto da área social, atrás apenas da Previdência.

Com respeito à descentralização, verifica-se que o MS vem direcionando cada vez mais recursos para os entes federados, buscando assim concretizar o pilar da descentralização do SUS. Do total do orçamento do Ministério da Saúde (MS) em 1995, 87\% dos recursos eram aplicados diretamente pelo ministério, já em 2010 foi de 31\%. Nesse caso, os estados e municípios passaram a absorver uma parcela maior do orçamento do MS. Os municípios passaram de 5\% (1995) para 43\% (2010), já os estados passaram de 6\% (1995) para 24\% (2010).

Alguns autores como Duarte et al. (2009) e Piola et al. (2013) fazem referência à falta de critérios de rateio e à inequidade nas transferências fundo a fundo do MS (terceiro fato estilizado do financiamento). Os questionamentos referem que os critérios de distribuição (hoje pautados basicamente pelo fator população ou per capita) não levam em consideração outros fatores importantes, como as condições estruturais e as condições de saúde de cada ente federado. Dessa maneira, percebe-se que alguns estados (que possuem piores indicadores de saúde) recebem em termos per capita uma quantidade menor de recursos do que estados em melhores condições. Outra situação que merece atenção é a discrepância no acesso da população aos serviços do SUS. Duarte et al. (2009) demonstram que pessoas mais pobres (pertencentes aos $1^{\circ}, 2^{\circ}$ e $3^{\circ}$ decis de renda) se beneficiam mais dos procedimentos de atenção básica, já a população mais rica (pertencentes aos $8^{\circ}, 9^{\circ} \mathrm{e}$ $10^{\circ}$ decis de renda) se beneficia mais dos serviços de MAC.

O quarto fato estilizado enfatiza um elemento da legislação do SUS, que implica constrangimentos ao financiamento da saúde pública, que se refere à permissão da exploração do setor de saúde ao segmento privado (sem a devida delimitação do tipo de exploração, bem como de sua regulação). Dessa maneira, Ocké-Reis (2009, p.8) afirma que "[...] o Estado não induziu a democratização das instituições que regulamentam os médicos liberais e os prestadores de hospitais privados".

Esse constrangimento do financiamento deve-se, conforme exposto por Piola et al. (2009), que o SUS compete com recursos destinados ao setor privado no formato de subsídios à população (deduções no imposto de renda) e financiamento de planos privados para servidores públicos. Ainda, para o autor, o 
sistema de saúde brasileiro, tanto em termos de oferta de serviços como em financiamento, pode ser considerado pluralista, pois contempla o SUS (financiado com recursos públicos), o segmento de seguros e planos de saúde privados (financiado pelo pré-pagamento de empresas e famílias com subsídios públicos), o segmento de atendimento a servidores públicos e militares (financiado em coparticipação de servidores e governo, mas provido amplamente pela rede privada) e o segmento exclusivamente privado, com acesso da população mediante pagamento no ato (out-of-pocket).

Nesses termos, percebe-se que, de um lado, tem-se a concorrência por recursos públicos entre o SUS e o segmento privado, de outro, há uma relativa falta de regulação sobre o setor privado, implicando maiores dificuldades para garantir um sistema de saúde público universal, como é o caso do SUS.

Por fim, tem-se o problema da má gestão dos recursos. Para o CONASS (2006; 2011), a má gestão passa pela questão da federalização e da precária qualificação do quadro de gestores dos sistemas de saúde. Em termos da federalização, a quantidade excessiva de pequenos municípios gera o problema de ineficiência de escala, ou seja, subutilização de equipamentos hospitalares e mau dimensionamento das necessidades de saúde da população, gerando despesas desnecessárias ou de baixo impacto custobenefício. Uma das formas que está sendo utilizada para lidar com essa questão é o processo de regionalização, que cria regiões de saúde, onde alguns municípios concentram a oferta de serviços de saúde mais especializados (MAC).

Com relação à qualificação dos gestores, o CONASS (2006; 2011) avalia que a gestão do sistema de saúde nos municípios necessita de quadros técnicos mais habilitados, com qualificações que vão além dos conhecimentos específicos da área da saúde, contemplando também uma visão holística do sistema. Pode-se incluir, também, que a falta de mecanismos de incentivos e de regulação das gestões cria maiores dificuldades para gerar ganhos de eficiência no sistema.

Em síntese, verifica-se que o financiamento do SUS possui sérias dificuldades, tanto em termos de volume de recursos como em termos de gestão do sistema. Essa realidade abre espaço para a manutenção de estudos que permitam ampliar a compreensão do sistema e propor a melhoria da política de saúde. 


\section{Metodologia}

Nesta seção, serão apresentadas as duas técnicas de análise multivariada empregadas para avaliar as relações entre despesas públicas e indicadores de saúde. Em um primeiro momento, apresenta-se a técnica de Análise Fatorial cujo objetivo será a construção de indicadores sintéticos para o grupo de variáveis da saúde, e, na sequência, a análise de Correlação Canônica, visando verificar a natureza do relacionamento entre esses dois grupos de variáveis.

\subsection{Análise Fatorial (AF)}

A AF é uma técnica estatística multivariada que tem por objetivo explicar a correlação entre um conjunto de " $p$ " variáveis observáveis em termos de " $m$ " fatores (ou constructos) não observáveis, responsáveis pela correlação observada dentro daquele conjunto. O primeiro passo em AF é verificar se a amostra é adequada ao emprego da técnica, uma vez que determinada estrutura de dados pode ser melhor representada de outra maneira ou com o emprego de outra metodologia. Duas técnicas são, em geral, empregadas quando se deseja verificar a adequacidade dos dados.

O teste de esfericidade de Bartlett (1954) testa a hipótese nula de que as variáveis não são correlacionadas na população $\left(\mathrm{H}_{0}: \rho=\mathrm{I}\right)^{7}$, ou seja, não se pode assumir qualquer relacionamento entre as variáveis, enquanto que a medida de Kaiser-Meyer-Olkin (KMO) calcula um índice baseado em regressões. Nesse caso, para valores entre 0.5 e 1.0, os dados são adequados à $\mathrm{AF}$, e para valores menores do que 0.5 , descarta-se essa possibilidade.

Segundo Johnson e Wichern (1988), o modelo fatorial ortogonal pode ser descrito da seguinte forma, em notação matricial:

$$
\mathbf{X}-\boldsymbol{\mu}=\mathrm{LF}+\boldsymbol{\varepsilon}
$$

em que $\mathbf{X}$ - $\boldsymbol{\mu}$ é o vetor coluna de "p" variáveis aleatórias centradas na média, L é a matriz "p x m" de pesos (ou carregamentos), F é o vetor coluna de " $\mathrm{m}$ " fatores comuns e $\boldsymbol{\varepsilon}$ é o vetor coluna de "p" erros aleatórios. Assume-se que $\mathrm{E}(\mathbf{F})=\mathbf{0}, \mathrm{E}(\boldsymbol{\varepsilon})$

\footnotetext{
${ }^{7}$ Em outras palavras, assume-se a hipótese de que a matriz de correlação populacional é a matriz Identidade, cujos elementos fora da diagonal principal são todos iguais a zero (correlação nula entre as variáveis).
} 
$=\mathbf{0}$ e $\operatorname{cov}(\boldsymbol{\varepsilon}, \mathbf{F})=0$. A AF visa aproximar a matriz de covariância " $\Sigma$ " de acordo com a seguinte estrutura de covariância:

$$
\Sigma=\operatorname{cov}(\mathbf{X})=\mathrm{LL}^{\prime}+\Psi \text { ou } V\left(\mathrm{X}_{\mathrm{i}}\right)=1_{\mathrm{i} 1}^{2}+1_{\mathrm{i} 2}^{2}+\ldots+1_{\mathrm{im}}^{2}+\Psi_{\mathrm{i}}
$$

em que a soma dos $l_{i m}^{2}$ elementos corresponde à comunalidade ou à porção da variância total explicada pelos "m" fatores comuns e $\Psi_{\mathrm{i}}$ a variância específica. De acordo com Hair Jr. et alli. (2005), o objetivo primaz da AF é estimar a matriz amostral de pesos, a partir da qual será possível chegar à estrutura de covariância desejada. Johnson e Wichern (1988) sugerem três possibilidades metodológicas para se estimar a matriz de pesos, quais sejam: 1) a Análise de Componentes Principais (ACP); 2) a Análise de Máxima Verossimilhança; e 3) os Mínimos Quadrados Ordinários. O presente trabalho utilizará a metodologia de estimação por meio das Componentes Principais ${ }^{8}$, uma vez que não se fazem maiores pressuposições acerca da distribuição dos dados e possibilita a redução da dimensionalidade da matriz amostral.

Seja $\mathrm{m}<\mathrm{p}$ o número de fatores comuns, a matriz de pesos "L" será estimada de acordo com a seguinte relação:

$$
\widehat{\mathrm{L}}=\widehat{\mathrm{C} \mathrm{D}_{\lambda}^{1 / 2}}
$$

em que $\widehat{\mathrm{C}}$ corresponde à matriz de autovetores de $\Sigma$ e $\mathrm{D}_{\lambda}^{1 / 2}$ à matriz diagonal raizquadrada dos autovalores associados. Para facilitar a interpretação dos fatores, Kaiser (1958) sugere o emprego da rotação VARIMAX, uma vez que permite obter uma estrutura de pesos mais simples tal que cada variável tenha um peso elevado em um único fator e pesos relativos menores nos demais fatores. Uma vez estimados os fatores, é conveniente estimar os escores fatoriais para cada observação individual $\mathbf{x}=\left[\begin{array}{llll}\mathrm{x}_{1} & \mathrm{x}_{2} & \ldots & \mathrm{x}_{\mathrm{p}}\end{array}\right]$ '. De acordo com Bartlett (1954), os escores fatoriais podem ser calculados por meio da matriz $\widehat{\mathbf{F}}=\left(\widehat{\mathrm{L}}^{\top} \widehat{\mathrm{L}}\right)^{-1} \mathrm{~L}^{\prime} \mathbf{Z}$, em que $Z$ são as variáveis originais padronizadas.

\footnotetext{
${ }^{8}$ Conforme Johnson e Wichern (1988), o método de ACP consiste em uma transformação nas variáveis originais em um novo conjunto de variáveis não correlacionadas, tendo propriedades especiais em termos de variância. As novas variáveis que são criadas pela ACP são combinações lineares das variáveis originais.
} 
Uma possível aplicação dos escores fatoriais relaciona-se à criação de indicadores sintéticos, tal que: INDICADOR $=\widehat{\mathrm{F}_{1}} \times \%$ VAREXPLICADA + $\mathrm{F}_{2} \mathrm{x} \%$ VAREXPLICADA, para $\mathrm{m}=2$ fatores. A construção de indicadores sintéticos auxilia no diagnóstico do problema bem como no estabelecimento de uma hierarquia entre as observações.

\subsection{Análise de Correlação Canônica (ACC)}

Para examinar a relação entre dois conjuntos distintos de variáveis, utilizase a ACC. Segundo Hair Jr. et alli. (2005), recomenda-se o uso da Correlação Canônica quando se têm presentes: (i) medidas de resultado ou desempenho; (ii) múltiplas variáveis dependentes e independentes; e (iii) um conceito mais amplo de variável dependente. A ACC foi desenvolvida por Hotelling (1936) e objetiva identificar e quantificar o poder de associação entre dois conjuntos de variáveis. De acordo com Johnson e Wichern (1988), o modelo de correlação canônica populacional pode ser descrito de acordo com a seguinte estrutura:

$$
\mathbf{X}=\left[\frac{\mathbf{X}_{\mathrm{p}}^{(1)}}{\mathbf{X}_{\mathrm{q}}^{(2)}}\right] \text { com média } \mu=E(\mathbf{X})=\left[\frac{\mu_{\mathrm{p}}^{(1)}}{\mu_{\mathrm{q}}^{(2)}}\right]
$$

E matriz de covariância:

$$
\boldsymbol{\Sigma}=\mathrm{E}(\mathbf{X}-\boldsymbol{\mu})(\mathbf{X}-\boldsymbol{\mu})^{\prime}\left[\begin{array}{ll}
\Sigma_{11} & \Sigma_{12} \\
\Sigma_{21} & \Sigma_{22}
\end{array}\right]
$$

em que $\mathbf{X}_{\mathbf{p}}^{(\mathbf{1})}$ e $\mathbf{X}_{\mathbf{q}}^{(\mathbf{2})}$ denotam os dois grupos “p” e “q” de variáveis e $\boldsymbol{\Sigma}$ a matriz de covariância, no qual $\Sigma_{11}$ e $\Sigma_{22}$ representam a covariância dentro dos grupos 1 e 2, respectivamente, e $\Sigma_{12}$ e $\Sigma_{21}$ a covariância entre as variáveis pertencentes aos grupos 1 e 2 .

A ideia central subjacente à técnica canônica consiste em construir variáveis que são combinações lineares das "p" e "q" variáveis de cada grupo, sendo que haverá $\min (\mathrm{p}, \mathrm{q})$ variáveis canônicas em determinada análise. Desse modo, as combinações lineares deverão satisfazer a condição de que cada par de variáveis canônicas maximize a correlação canônica $\rho^{*}$. Portanto, o primeiro par de variáveis canônicas amostrais será o par de combinações lineares ${\widehat{U_{1}}}_{1}=\mathbf{a}^{\prime} \mathbf{X}^{(\mathbf{1})}$ e 
$\widehat{U}_{2}=\mathbf{b}^{\prime} \mathbf{X}^{(2)}$. Os vetores a' e b' deverão ser tais que maximizem a correlação entre $\mathrm{U}$ e $\mathrm{V}-\rho_{1}^{*}$. O segundo par de variáveis canônicas deverá maximizar a correlação entre $\mathrm{U}$ e $\mathrm{V}-\rho_{2}^{*}$ sujeito à condição de não ser correlacionado ao primeiro par e assim por diante.

Apesar de as variáveis canônicas serem variáveis artificiais, Johnson e Wichern (1988) argumentam que, para se identificar a dimensão dessas variáveis, é necessário verificar a correlação entre as variáveis canônicas e as variáveis originais, uma vez que o resultado dessa métrica fornece uma avaliação de como cada variável original contribui para dada variável canônica e facilita a interpretação dos resultados. Para variáveis originais não padronizadas, têm-se:

$$
\begin{aligned}
& \mathrm{R}_{\mathbf{U}, \mathbf{X}^{(\mathbf{1})}}=\widehat{\mathrm{AS}}_{11 \mathrm{D}_{11}}-\frac{1}{2} \\
& \mathrm{R}_{\mathbf{V}, \mathbf{X}^{(2)}}=\widehat{\mathrm{BS}}_{22 \mathrm{D}_{22}}^{-\frac{1}{2}} \\
& \mathrm{R}_{\mathbf{U}, \mathbf{X}^{(2)}}=\widehat{\mathrm{A}} \mathrm{S}_{12 \mathrm{D}_{22}}^{-\frac{1}{2}} \\
& \mathrm{R}_{\mathbf{V}, \mathbf{X}^{(\mathbf{1})}}=\widehat{B} \mathrm{~S}_{21 \mathrm{D}_{11}^{-\frac{1}{2}}}
\end{aligned}
$$

em que $\mathrm{D}_{11}^{-\frac{1}{2}}$ é a matriz diagonal (pxp) cujo i-ésimo elemento da diagonal principal corresponde a $\mathrm{V}\left(\mathrm{x}_{\mathrm{i}}^{(1)}\right)^{-1 / 2}$, e $\mathrm{D}_{22}^{-\frac{1}{2}}$ a matriz diagonal (qxq) cujo i-ésimo elemento corresponda a $\mathrm{V}\left(\mathrm{x}_{\mathrm{i}}^{(2)}\right)^{-1 / 2}$.

\section{Dados e análise dos resultados}

\subsection{Base de dados}

Para a construção do modelo de Correlação Canônica, foram utilizados dois grupos de variáveis de natureza distinta e que se supõe correlacionados. Os dados foram selecionados para os 26 estados da Federação mais o Distrito Federal, tendo como referência o ano de 2010. A escolha desse ano decorre da disponibilidade de dados para todas as variáveis envolvidas e para todas as unidades da federação. 
O primeiro grupo de variáveis é formado por atributos de saúde extraídos do banco de dados DATASUS ${ }^{9}$. Esses atributos estão disponibilizados na forma de indicadores e dados básicos de saúde e estão dispostos em sete grupos, de acordo com a seguinte classificação: a) indicadores demográficos (54 indicadores); b) indicadores socioeconômicos (24 indicadores); c) indicadores de mortalidade (59 indicadores); d) indicadores de morbidade (76 indicadores); e) indicadores de recursos (95 indicadores); f) indicadores de cobertura (45 indicadores); e g) indicadores de fatores de risco e proteção (43 indicadores). Desse conjunto, apenas o grupo $\mathrm{G}$ não foi utilizado na análise, uma vez que a disponibilidade de dados era incompleta para todas as unidades da federação.

O segundo grupo de variáveis refere-se às despesas governamentais por função, denominadas aqui de variáveis fiscais, extraídas do sítio da Secretaria do Tesouro Nacional no item Execução Orçamentária ${ }^{10}$. Esse conjunto de dados foi arbitrado de acordo com a característica de sua dimensão, ou seja, em que medida uma dada variável fiscal poderia impactar o bem-estar relacionado à saúde de um indivíduo, conforme apontado pela literatura dos determinantes sociais da saúde, apontado na seção 2. Nesse sentido, foram selecionadas as variáveis por função: i) assistência social e previdência; ii) saúde; iii) saneamento; iv) educação e cultura; v) gestão ambiental; e vi) esportes e lazer.

As variáveis fiscais receberam os seguintes tratamentos para serem trabalhadas em termos de indicadores fiscais: i) as despesas por função foram divididas pelo PIB estadual para o período de 2002 a 2010. Essa transformação possibilitou padronizar as variáveis entre os estados bem como demonstrar uma medida do esforço monetário realizado pelo estado para cada função social; ii) foi considerada a média das despesas por função/PIB do período. O objetivo dessa transformação foi tentar capturar os possíveis impactos de longo prazo dessas variáveis sobre os indicadores de saúde; iii) foram linearizadas objetivando a correção de problemas de normalidade devido à presença de outliers.

9 Departamento de Dados do Sistema Único de Saúde (DATASUS). Disponível em: http://tabnet.datasus.gov.br/cgi/idb2012/matriz.htm.

${ }^{10}$ Disponível em: http://www.tesouro.fazenda.gov.br/. 


\subsection{Construção dos indicadores sintéticos da saúde}

Os indicadores sintéticos foram construídos através da metodologia de AF para o primeiro grupo de variáveis, isto é, os seis grupos de indicadores de saúde disponibilizados pelo DATASUS (2015). Como primeiro passo, foi utilizado a ACP para excluir variáveis redundantes. Na análise empírica, é comum deparar-se com problemas de ajuste do modelo $\mathrm{Y}=\mathrm{X} \boldsymbol{\beta}+\boldsymbol{\varepsilon}$ à amostra multivariada, uma vez que o conjunto de dados geralmente apresenta um número expressivo de observações e variáveis (nxp). Ocorre que as linhas da matriz $X_{(n x p)}$ podem ser aproximadamente colineares gerando problemas de inconsistência e variáveis redundantes que pouco contribuem com a análise.

Segundo Johnson e Wichern (1992), o método da ACP pode ser empregado com o objetivo de reduzir a dimensionalidade da matriz de dados amostrais bem como para a exclusão de variáveis redundantes. $\mathrm{O}$ algoritmo é bastante simples, uma vez que se devem excluir as variáveis cujos autovalores são inferiores a 0.7. Utilizou-se o pacote estatístico MATLAB para toda a análise. Empregando o algoritmo de exclusão de variáveis redundantes, foram excluídas 48 variáveis do grupo "a", 18 variáveis do grupo "b", 49 variáveis do grupo "c", 56 do grupo "d", 75 variáveis do grupo “e” e, por fim, 34 variáveis do grupo "f”"11.

O próximo passo foi verificar, por meio dos testes de esfericidade de Bartlett e KMO, se os dados de saúde são adequados à AF. O mesmo procedimento não foi empregado para as variáveis fiscais, em virtude da não realização de AF para essas variáveis. A Tabela 2 resume os resultados por grupos de variáveis de saúde.

\section{Tabela 2 - Testes de esfericidade de Bartlett e KMO (variáveis de saúde)}

\begin{tabular}{|c|c|c|c|c|c|c}
\hline Teste & Demográfico & Socioeconômico & Mortalidade & Morbidade & Recursos & Cobertura \\
\hline Bartlett & RejeitaHo & RejeitaHo & RejeitaHo & RejeitaHo & RejeitaHo & RejeitaHo \\
\hline KMO & 0.697 & $0.48^{*}$ & 0.56 & 0.53 & 0.57 & $0.40^{*}$ \\
\hline
\end{tabular}

Fonte: dados da pesquisa.

De acordo com a Tabela 2, os dados de saúde são, em geral, adequados à AF, à exceção das variáveis "socioeconômica" e de "cobertura", que apresentaram um índice KMO inferior a 0.5. Entretanto, optou-se por não excluir essas variáveis

\footnotetext{
${ }^{11}$ As variáveis fiscais não foram submetidas ao algoritmo de exclusão de variáveis tendo em vista que já são disponibilizadas sob a forma de indicadores, não sendo necessário o emprego da $\mathrm{AF}$ a esses dados.
} 
por dois motivos: primeiro, os valores da estatística KMO não são tão baixos a ponto de indicar total rejeição dessas variáveis; segundo, o teste de esfericidade de Bartlett rejeitou a hipótese nula de ausência de correlação populacional ( $\rho=I)$, contrariando a estatística KMO.

Uma vez adequada a estrutura de dados, realizou-se a AF da nova matriz. Foram utilizados dois critérios para a definição do número de fatores. $\mathrm{O}$ critério de Kaiser considera o número de fatores " $\mathrm{m}$ ” como sendo igual ao número de autovalores maiores do que 1, enquanto que o critério da variância explicada leva em consideração o percentual de variância explicada acima de $70 \%$. A Tabela 3 resume os resultados para o conjunto de variáveis de saúde, após a rotação VARIMAX.

Tabela 3 - Número de fatores segundo o critério de Kaiser

\begin{tabular}{|c|c|c}
\hline Variáveis & Número de fatores & Variância Explicada \\
\hline Demografia & 2 fatores & $81.30 \%$ \\
\hline Socioeconômico & 2 fatores & $82.30 \%$ \\
\hline Mortalidade & 4 fatores & $81 \%$ \\
\hline Morbidade & 6 fatores & $80.74 \%$ \\
\hline Recursos & 6 fatores & $85.83 \%$ \\
\hline Cobertura & 4 fatores & $73.81 \%$ \\
\hline
\end{tabular}

Fonte: dados da pesquisa.

A variável "demografia" pode ser representada por dois fatores. O primeiro fator $\left(F_{1 d}\right)$ do grupo demografia é melhor representado pela: (i) população total ($0.5426)^{12}$; (ii) taxa bruta de mortalidade (-0.9585); (iii) a razão de dependência de idosos na população (0.7371); e (iv) a razão de óbitos totais (-0.9620). O F 1 d significará "exposição de risco de vida da população". O segundo fator $\left(\mathrm{F}_{2 \mathrm{~d}}\right)$ do grupo demografia é representado pela: (i) esperança de vida da mulher aos 60 anos (0.7827); e (ii) proporção de menores de 5 anos de idade na população (0.9347). O F $F_{2 d}$ significará "qualidade de vida das populações mais sensíveis".

No grupo "socioeconômico", foram delimitados dois fatores. O primeiro fator $\left(F_{1 s e}\right)$, denominado "renda da população" é representado pelo: (i) PIB per capita (0.7103); (ii) Índice de Gini da renda domiciliar per capita (-0.8262); (iii)

\footnotetext{
${ }^{12}$ Os valores entre parênteses representam a correlação entre o fator e a respectiva variável. Desse modo, quanto maior a correlação entre o fator e uma determinada variável, mais representativa será esta variável para aquele fator.
} 
proporção de pessoas de baixa renda - renda domiciliar per capita menor do que 1/4 do salário mínimo (-0.9856); e (iv) proporção de crianças em situação domiciliar de baixa renda - renda domiciliar per capita menor do que $1 / 2$ do salário mínimo (0.9810). O Fator $2\left(\mathrm{~F}_{2 \mathrm{se}}\right)$, por sua vez, é composto pela: (i) escolaridade da população de 15 anos com 4 a 7 anos de estudo (0.8193); e (ii) taxa de trabalho infantil (0.7267). O F 2se significará "escolaridade".

No grupo "mortalidade", foram construídos quatro fatores. O primeiro fator $\left(F_{1 \mathrm{mt}}\right)$ é representado pela: (i) taxa de mortalidade infantil neonatal precoce (0.9016); (ii) taxa de mortalidade infantil pós-neonatal (0.8019); (iii) taxa de mortalidade por neoplasias (-0.8615); e (iv) taxa de mortalidade por doenças transmissíveis (-0.7146). O segundo fator $\left(\mathrm{F}_{2 \mathrm{mt}}\right)$ é composto por: (i) mortalidade de causas definidas (0.8246); e (ii) mortalidade diabetes masculina (0.9095). O terceiro fator $\left(\mathrm{F}_{3 \mathrm{mt}}\right)$ é formado pela: (i) taxa de mortalidade por causas externas ($0.9204)$; e (ii) taxa de mortalidade por acidente de trabalho (-0.7035). Por fim, o quarto fator $\left(\mathrm{F}_{3 \mathrm{mt}}\right)$ é representado pelos: (i) óbitos de morte materna (0.8592); e (ii) taxa de mortalidade por doenças do aparelho circulatório (-0.6686).

No grupo "morbidade", foram definidos seis fatores. O primeiro fator $\left(\mathrm{F}_{1 \mathrm{md}}\right)$ é representado pelas doenças: (i) coqueluche (-0.8405); (ii) câncer masculino de estômago (-0.5522); (iii) contaminação HIV via sexual (0.5358); (iv) internação por câncer maligno (-0.8652); (v) internação por hipertensão (-0.8609); e (vi) internação por problemas cardiovasculares (-0.6850). O F $1 \mathrm{md}$ será chamado de fator de "taxa de internação por doenças crônicas", uma vez que as maiores correlações são (iv) e (v). O fator $2\left(\mathrm{~F}_{2 \mathrm{md}}\right)$ é representado pelas variáveis: (i) internação diabete melito (-0.8621); (ii) internação vascular cérebro (-0.8196); (iii) internação pneumonia (-0.6911); (iv) internação atenção básica (-0.9184); e (v) internação causas externas (-0.5971). Como as variáveis que apresentam maior correlação com o fator são (i), (ii) e (iv), o $\mathrm{F}_{2 \mathrm{md}}$ será chamado de fator de "internação por aterosclerose". O fator $3\left(\mathrm{~F}_{3 \mathrm{md}}\right)$, por sua vez, é representado pelas variáveis: (i) outros cânceres de pele (-0.6016); e (ii) internações por acidentes de transporte (0.7711). O fator $F_{3 m d}$ será chamado de fator de "acidentes de transporte". O fator $4\left(\mathrm{~F}_{4 \mathrm{md}}\right)$ é representado pelas variáveis: (i) hepatite $\mathrm{B}(0.8979)$; (ii) sífilis congênita (0.6611); (iii) meningite (0.9579); e (iv) leptospirose (0.9013). Como as maiores correlações são das variáveis (i), (iii) e (iv), o fator será chamado de fator de "incidência de doenças transmissíveis". O fator $5\left(\mathrm{~F}_{5 \mathrm{md}}\right)$ é representado pelas 
variáveis: (i) acidentes e doenças de trabalho (0.8019); e (ii) acidentes sem registro (0.9014). Esse fator pode ser chamado de fator de "acidentes de trabalho". Por fim, o fator $6\left(\mathrm{~F}_{6 \mathrm{md}}\right)$ é representado por apenas uma variável, internação por violência legal (-.09238). $\mathrm{O}$ fator $\mathrm{F}_{6 \mathrm{md}}$ receberá o mesmo nome da variável.

No grupo "recursos", foram selecionados seis fatores. O primeiro fator $\left(\mathrm{F}_{1 \mathrm{r}}\right)$ é composto por: (i) profissionais de odontologia (0.9071); (ii) profissionais de enfermagem (0.5859); (iii) profissionais da nutrição (0.7847); (iv) profissionais da veterinária (0.5801); (v) postos de trabalho privados sem fins lucrativos (0.7561); (vi) número de equipamentos de ressonância (0.9380); (vii) número de equipamentos de tomografia (0.8991); (viii) número de equipamentos de ultrassonografia (0.8361); e (ix) gastos em ações de serviços públicos (-0.8007). Como as variáveis que apresentam a maior correlação são (i), (vi) e (vii), o fator $\mathrm{F}_{1 \mathrm{r}}$ será chamado de fator de "estrutura técnica especializada". $\mathrm{O}$ fator $2\left(\mathrm{~F}_{2 \mathrm{r}}\right)$, por sua vez, é bem representado pelas variáveis: (i) postos de trabalho em nível superior (0.8804); (ii) postos de trabalho em instituições privadas sem fins lucrativos (-0.5769); (iii) postos de trabalho na área de enfermagem (0.6545); e (iv) número de leitos públicos (0.8918). $\mathrm{O}$ fator $\mathrm{F}_{2 \mathrm{r}}$ pode ser chamado de fator de "postos de trabalho em nível superior", uma vez que as variáveis que apresentaram maior correlação foram (i) e (iv). O fator $3\left(\mathrm{~F}_{3 \mathrm{r}}\right)$ é representado pela variável, leitos no SUS municipais (-0.9543), podendo ser chamada de fator "leito SUS municipal”. O fator $4\left(\mathrm{~F}_{4 \mathrm{r}}\right)$ é composto pela variável, gastos familiares em saúde (outros) (0.8785) e será chamado fator de "outros gastos familiares". O fator $5\left(\mathrm{~F}_{5 \mathrm{r}}\right)$ é representado pelas variáveis: (i) profissionais de auxiliar de enfermagem (0.8020); e (ii) gastos familiares em planos de saúde (0.7337). Pode-se chamá-la de fator de "renda de planos de saúde". Por fim, o fator $6\left(\mathrm{~F}_{6 r}\right)$ é representado pela variável, postos de trabalho em nível superior federal (0.8930), podendo ser chamada de fator de "postos de trabalho federais".

No grupo "cobertura", foram delimitados quatro fatores. O primeiro fator $\left(F_{1 c}\right)$ é representado pelas variáveis: (i) assistência médica ambulatorial (-0.6354); (ii) assistência odontológica (-0.6780); (iii) abastecimento urbano de água (0.7219); e (iv) esgotamento urbano (-0.8843). Tendo em vista que as maiores correlações são das variáveis (iii) e (iv), o $F_{1 c}$ será chamado de fator de "abastecimento e esgotamento urbano". O segundo fator $\left(\mathrm{F}_{2 \mathrm{c}}\right)$ é composto pelas variáveis: (i) diagnóstico patologia clínica (-0.8781); (ii) partos hospitalares 
(0.5833); e (iii) crianças vacinadas contra BCG (-0.7321). As variáveis que apresentam a maior correlação são (i) e (iii). Portanto, o $F_{2 c}$ será chamado de fator de "eficiência hospitalar". O fator $3\left(\mathrm{~F}_{3 \mathrm{c}}\right)$ é composto pelas variáveis: (i) vacina tetravalente (0.8728); e (ii) tríplice viral (0.9335). $\mathrm{O} \mathrm{F}_{3 \mathrm{c}}$ será chamado de fator de "vacinação em idade recomendada". Por fim, o fator $4\left(\mathrm{~F}_{4 \mathrm{c}}\right)$ é representado pelas variáveis: (i) internação 12 meses (-0.6851); e (ii) coleta de lixo urbano (0.6784), podendo se chamar de fator de "infraestrutura urbana".

A construção de fatores possibilita uma interpretação mais adequada quando se trabalha com grandes bancos de dados. Diante do resultado fatorial, há duas possibilidades com que se trabalhar: primeiro, com os seis grupos de variáveis, levando em consideração o número de fatores sugerido pelo critério de Kaiser, em cada grupo, ou seja, trabalhar-se-iam com 24 variáveis (ou fatores); ou, em segundo lugar, construir um indicador sintético em cada grupo, totalizando seis indicadores de saúde.

O indicador sintético é um resumo do comportamento de todas as variáveis (ou fatores) dentro daquele grupo. Por exemplo, considerando o grupo de variáveis de morbidade, o indicador geral "morbidade" irá capturar o efeito de todos os seis fatores de uma só vez. A construção do indicador sintético é realizada através dos coeficientes dos escores fatoriais dados pela AF, conforme exposto na seção 3 . Sendo assim, optou-se por trabalhar com apenas o indicador sintético de cada grupo para fins de análise de correlação canônica. Esse procedimento facilitará a interpretação do efeito das variáveis fiscais sobre as variáveis de saúde.

Os seis indicadores sintéticos estão expostos na Tabela 7, no Apêndice, adotando a nomenclatura geral: (i) demográfico; (ii) socioeconômico; (iii) mortalidade; (iv) morbidade; (v) recursos; e (vi) cobertura.

\subsection{Análise da correlação canônica}

Tendo a base de dados a ser trabalhada, seis indicadores sintéticos da saúde e os seis indicadores fiscais, procede-se à análise dos resultados da ACC. Uma vez sendo uma técnica de dependência, a ACC estudará o efeito do conjunto de variáveis fiscais sobre o conjunto de variáveis da saúde.

A Tabela 4 apresenta o valor da correlação canônica para cada par de variáveis canônicas. O primeiro par de variáveis canônicas obviamente apresentou 
a mais elevada correlação (0.9531), seguido do segundo par (0.7880), terceiro par (0.7440) e quarto par (0.6910). O quinto e sexto pares, por apresentarem baixa correlação, serão desconsiderados da análise.

\section{Tabela 4 - Correlações canônicas amostrais e coeficientes das variáveis canônicas amostrais}

\begin{tabular}{|c|c|c|c|c|c|c|c|c|c|c|c|c|c|c|}
\hline & \multicolumn{6}{|c|}{ coeficientes variáveis canônicas saúde } & \multirow[b]{2}{*}{$\rho^{*}$} & & \multicolumn{6}{|c|}{ coeficientes variáveis canônicas fiscais } \\
\hline & $z_{1}^{(1)}$ & $z_{2}^{(1)}$ & $z_{3}^{(1)}$ & $\mathrm{z}_{4}^{(1)}$ & $z_{5}^{(1)}$ & $z_{6}^{(1)}$ & & & $z_{1}^{(2)}$ & $\mathrm{z}_{2}^{(2)}$ & $z_{3}^{(2)}$ & $\mathrm{z}_{4}^{(2)}$ & $z_{5}^{(2)}$ & $z_{6}^{(2)}$ \\
\hline$a_{1}^{\prime}$ & -0.0917 & 0.9298 & -0.1825 & 0.1126 & -0.0304 & -0.1693 & 0.95 & $b_{1}^{\prime}$ & 0.1456 & 0.4349 & -0.1034 & 0.5355 & 0.1326 & 0.2645 \\
\hline$a_{2}^{\prime}$ & 0.7121 & 0.2871 & 0.1514 & 0.3438 & 0.4896 & -0.0455 & 0.79 & $b_{2}^{\prime}$ & -0.7702 & 0.0589 & -0.2787 & 0.0080 & -0.0138 & 0.5857 \\
\hline$a_{3}^{\prime}$ & -0.7349 & -0.0180 & -0.0184 & 0.6508 & 0.9555 & 0.7549 & 0.74 & $b_{3}^{\prime}$ & 0.5573 & 0.0047 & 0.3157 & -0.1536 & -0.9424 & 0.4140 \\
\hline$a_{4}^{\prime}$ & 0.2889 & 0.8579 & 1.2121 & -0.2489 & 0.2480 & 0.7401 & 0.69 & $b_{4}^{\prime}$ & 0.0601 & 0.8363 & 0.1886 & -1.0237 & 0.2273 & -0.1441 \\
\hline$a_{5}^{\prime}$ & 0.4083 & 0.0440 & -0.8238 & 0.7553 & -0.9269 & -0.0377 & 0.07 & $b_{5}^{\prime}$ & 0.4103 & 0.1827 & -1.1111 & -0.2569 & 0.5179 & 0.8465 \\
\hline$a_{6}^{\prime}$ & -0.0574 & -1.4164 & -1.1497 & -0.6869 & 0.1228 & 0.6311 & 0.04 & $b_{6}^{\prime}$ & -0.1834 & 1.8073 & -1.5581 & 0.1447 & -0.3231 & -0.4858 \\
\hline
\end{tabular}

Fonte: dados da pesquisa. *correlação canônica.

Dadas as fortes correlações entre os indicadores fiscais e indicadores de saúde, sugere-se o efeito positivo dos indicadores fiscais sobre os indicadores da saúde. A Tabela 4 também apresenta os coeficientes das variáveis canônicas da saúde e fiscais $\left(\mathrm{z}_{\mathrm{i}}{ }^{(1)} \mathrm{e} \mathrm{z}_{\mathrm{j}}{ }^{(2)}\right.$, respectivamente). Através desses coeficientes é possível obter os quatro pares de variáveis canônicas mais representativos (a' ${ }_{1}$ e b' ${ }_{1}$; ${ }^{\prime}{ }_{2} \mathrm{e}$ $b_{2}$; a' ${ }_{3}$ e b' ${ }_{3}$; a' ${ }_{4}$ e b' ${ }_{4}$ ) os quais denotam o par de combinações lineares, a' ${ }_{i}$ e b' que maximizam a correlação canônica.

Na sequência são apresentados outros testes de significância. Segundo Hair Jr. et alli (2005), afora as correlações de pequena magnitude, três outros testes podem ser empreendidos se o objetivo é obter fundamentos ao excluir as correlações que parecem sem significado, quais sejam: (i) lambda de Wilks; (ii) teste $\chi^{2}$ (qui-quadrado) de Bartlett; e (iii) teste $\mathrm{F}$ de $\mathrm{Rao}^{13}$.

O lambda de Wilks é a versão generalizada do teste $\mathrm{F}$ da Análise de Variância (ANOVA). O objetivo é construir uma Análise de Variância Multivariada (MANOVA) e investigar se as distintas populações possuem o mesmo vetor médio, ou seja, assume-se, através da hipótese nula, que os vetores médios populacionais são todos iguais. Caso a hipótese nula seja rejeitada, podese concluir que pelo menos uma média difere das demais.

\footnotetext{
${ }^{13}$ Para uma maior compreensão desses testes, vide Hair Jr. et. alli. (2005) e Johnson e Wichern (1988).
} 
O teste $\chi^{2}$ de Bartlett, por sua vez, testa a inexistência de um relacionamento entre dois ou mais conjuntos de variáveis. A hipótese nula a ser testada é se dois ou mais grupos apresentam variâncias idênticas $\left(\sigma_{1}^{2}=\sigma_{2}^{2}=\sigma_{\mathrm{k}}^{2}\right)$. Caso contrário, haverá pelo menos um par de variâncias que difere entre si. O teste de Bartlett pode ainda ser utilizado para verificar a significância estatística individual das correlações canônicas.

Por fim, o teste $\mathrm{F}$ de Rao se assemelha muito ao teste $\chi^{2}$ de Bartlett. Ele testa a significância da relação entre dois ou mais grupos de variáveis. A rejeição da hipótese nula significará que existe significância entre os conjuntos de variáveis.

Tabela 5 - Testes estatísticos para as correlações canônicas

\begin{tabular}{c|c|c|c|c}
\hline \multirow{2}{*}{ MANOVA } & Lambda de Wilks & Estatística do Teste & Valor Crítico & $\mathrm{p}$ valor \\
& 0.0112 & 688.5089 & & 0 \\
\hline \multirow{2}{*}{$\mathbf{X}^{2}$ de Bartlett } & Valor de V & Estatística do Teste & Valor Crítico & $\mathrm{p}$ valor \\
\cline { 2 - 5 } & 87.5909 & 87.5909 & 23.2686 & 0 \\
\hline \multirow{2}{*}{ F de Rao } & Valor de F & Estatística do Teste & Valor Crítico & $\mathrm{p}$ valor \\
\cline { 2 - 5 } & 3.3952 & 3.3952 & 1.5886 & 0 \\
\hline
\end{tabular}

Fonte: dados da pesquisa.

A Tabela 5 apresenta o resumo desses testes. O lambda de Wilks apresentou um valor bem pequeno (0.0112), enquanto que a estatística $F$ do teste apresentou um valor bem elevado (688.50). Isso significa que a hipótese nula pode ser rejeitada com segurança, indicando que há pelo menos um vetor médio que difere dos demais. O teste geral de Bartlett, por sua vez, apresentou um valor "V" elevado (87.59). Como o "V" calculado foi maior que o "V" tabelado (23.26), pode-se rejeitar a hipótese nula e concluir que existe um relacionamento entre os dois conjuntos de variáveis. $\mathrm{O}$ teste $\mathrm{F}$ de Rao também indicou significância estatística entre os dois conjuntos de variáveis. O F calculado (3.39) foi maior que o F crítico (1.58) e a hipótese nula pode ser rejeitada, com segurança, portanto.

Por fim, é importante testar a significância individual de cada correlação canônica " $\rho$ ". A Tabela 6 apresenta os resultados individuais do teste. O primeiro par de variáveis canônicas é significativo por conta do primeiro teste de Bartlett já apresentado. Excluindo-se o primeiro par, faz-se o mesmo teste para as demais correlações canônicas e assim sucessivamente. Os resultados são os seguintes: o segundo par de variáveis canônicas é igual significativo (49.85 > 24.33); o terceiro 
par é significativo $(31.43>15.33)$; o quarto par é também significativo $(14.75>$ $8.34)$, enquanto que o quinto par não o é $(0.15<3.35)$. Desse modo, pode-se excluir com segurança as duas últimas correlações canônicas.

Tabela 6 - Testes de significância individual das correlações canônicas

\begin{tabular}{|l|c|c|c}
\hline & Correlação Canônica & Chi2 & Chi2 Crítico \\
\hline $1^{\mathrm{a}}$ correlação canônica & 0.9531 & 35.3356 & 23.2686 \\
\hline $2^{\mathrm{a}}$ correlação canônica & 0.7880 & 49.8588 & 24.3366 \\
\hline $3^{\mathrm{a}}$ correlação canônica & 0.7440 & 31.4369 & 15.3385 \\
\hline $4^{\mathrm{a}}$ correlação canônica & 0.6910 & 14.7520 & 8.3428 \\
\hline $5^{\mathrm{a}}$ correlação canônica & 0.0678 & 0.1530 & 3.3567 \\
\hline $6^{\mathrm{a}}$ correlação canônica & 0.0436 & 0.0000 & 0.0000 \\
\hline Foñ
\end{tabular}

Fonte: dados da pesquisa.

Sabendo que as duas últimas correlações guardam pouca informação sobre o relacionamento entre os conjuntos, procede-se à análise da matriz de correlação das variáveis canônicas com as variáveis originais (dispostas nas Tabelas 9 e 10 do Apêndice). A construção dessas matrizes nos auxilia na interpretação final dos resultados. Desse modo, pode-se inferir que a dimensão da variável canônica $\mathrm{U}_{1}$ (representante dos indicadores de saúde) é melhor representada pelos atributos relacionados às características "socioeconômica", "mortalidade" e "recursos". O atributo "socioeconômico" apresentou correlação positiva, enquanto que os outros dois atributos, as correlações apresentaram sinal negativo.

Esses resultados possibilitam avaliar que o primeiro grupo de variáveis pode ser considerado uma medida de "bem-estar privado", pois à medida que as condições socioeconômicas melhoram e os indicadores de mortalidade caem, observa-se uma ampliação do bem-estar da população. Por outro lado, o sinal negativo para a variável "recursos" pode estar considerando o maior peso dos gastos privados das famílias em saúde comparado com o esforço do gasto público. Nesse caso, o maior gasto privado estaria compensando a insuficiência de recursos públicos para a saúde. Por outro lado, a variável canônica $U_{1}$ apresentou elevada correlação com as variáveis "gestão ambiental", "saúde", "assistência e previdência" e "educação". Exceto para a gestão ambiental, as demais variáveis apresentaram correlação positiva.

Portanto, pode-se apontar que melhorias nas condições socioeconômicas da população (redução da taxa de trabalho infantil e da pobreza, aumento da renda per capita domiciliar e melhoras nos índices de escolaridade), redução na mortalidade 
(por neoplasias, doenças infecciosas e parasitárias, causas externas e taxa de mortalidade infantil) e recursos (aumento de profissionais na área de saúde, por exemplo) estão fortemente correlacionados aos gastos nas áreas de saúde, assistência social e previdência e educação. Por outro lado, as despesas em gestão ambiental as quais contemplam: preservação e conservação ambiental, controle ambiental, recuperação de áreas degradadas, recursos hídricos e meteorologia - apresentaram correlação negativa com os indicadores de saúde. É possível que essa correlação esteja relacionada mais fortemente aos indicadores de mortalidade e de morbidade.

\section{Conclusão}

O presente trabalho teve como principal objetivo avaliar a relação entre um conjunto de despesas públicas estaduais, pertencentes ao grupo dos determinantes sociais da saúde, e indicadores de saúde. A relação foi avaliada através da metodologia de análise multivariada de correlação canônica, resultando em uma correlação de 95,31\% entre o primeiro par de variáveis canônicas. Essa dependência mostrou-se significativa do ponto de vista estatístico passando pelo crivo dos testes multivariados de significância.

Uma segunda contribuição do trabalho foi a construção de seis indicadores sintéticos de saúde através da Análise Fatorial. A construção dos indicadores sintéticos de saúde possibilitou uma análise mais ampla, uma vez que capturou conjuntamente características que, isoladamente, não possibilitariam o mesmo resultado.

Destaca-se que a elevada correlação canônica foi reforçada pelo resultado das cargas canônicas, isto é, da correlação entre a variável canônica da saúde $\left(\mathrm{U}_{1}\right)$ e as variáveis originais. Nesses termos, a correlação entre a variável canônica e os indicadores de saúde demonstrou que os indicadores "socioeconômico", "mortalidade" e de "recursos" apresentaram as maiores correlações. Dentre estes resultados o que mais chamou a atenção foi a correlação negativa para o indicador recursos, indicando uma insuficiência de recursos públicos para área de saúde, tendo que ser compensado pelo aumento dos gastos privados das famílias em saúde.

Já a correlação entre a variável canônica e os indicadores fiscais apresentou maior correlação positiva para as despesas em saúde, assistência e previdência e educação. Essas correlações reforçam as hipóteses levantadas pela literatura da economia da saúde, ou seja, as condições de saúde da população dependem de um 
conjunto amplo de insumos, como, por exemplo: educação, hábitos de vida saudáveis de vida, como exercícios físicos, boa nutrição, e assistência à saúde.

Nesse particular, os resultados deste trabalho apontam que esse conjunto de insumos pró-saúde podem ser alcançados e/ou estimulados através do desenho de políticas públicas articuladas entre diferentes áreas (ou diferentes secretarias estaduais) para atingir objetivos conjuntos, abrindo um leque adicional de instrumentos que podem ser utilizados para promover a saúde nos estados brasileiros.

A proposição de políticas conjuntas ganha maior relevância na medida em que os estados são importantes atores na condução e articulação de políticas públicas nacionais, como é o próprio caso do SUS, da assistência social e da educação (FUNDEB, PNAE). Nesse sentido, os estados seriam os atores com melhores condições de coordenar e articular ações entre diferentes áreas para a promoção de objetivos conjuntos.

Outro argumento que reforça a necessidade de se desenhar políticas públicas articuladas é o apelo pela eficiência dos gastos, os ganhos de economias de escala e de escopo gerados. Por exemplo, políticas de assistência social e de educação que visem melhorar os insumos de saúde e hábitos de famílias e crianças podem melhorar as condições de saúde da população, reduzindo a demanda pela assistência de saúde, que por sua vez é um insumo mais caro.

Por fim, deve-se mencionar que a análise de correlação canônica é uma técnica geral de dependência geralmente empregada em situações nas quais estão presentes conceitos mais amplos de variáveis, como é o caso do trabalho em questão. Foi utilizado um conceito amplo de variáveis da saúde concomitante ao uso de um conceito amplo de gastos fiscais e a análise de correlação canônica é a única técnica disponível que captura esse relacionamento conjunto entre grupo de variáveis.

Para reforçar e complementar os resultados deste estudo, sugere-se que os resultados da correlação canônica sejam ampliados e aprimorados através de estudos econométricos que possibilitem avaliar os efeitos (coeficientes) individuais de cada despesa sobre algum indicador de saúde.

\section{Referências}

ARORA, S. Health, human productivity, and long-term economic growth. The Journal of Economic History, v. 61, n. 3, p. 699-749, 2001. 
BARTLETT, M. S. A note on multiplying factors for various chi-squared approximations. Journal of the Royal Statistical Society, v. 16, n. 2, p. 296-298, 1954.

BHARGAVA, A. et al. Modelling the effects of health on economic growth. Journal of Health Economics, v. 20, n. 3, p. 423-440, 2001.

BLOOM, D. E. et al. The effect of health on economic growth: a production function approach. World Development, v. 32, n. 1, p. 1-13, 2004.

BLOOM, D. E.; CANNING, D. Health and economic growth: reconciling the micro and macro evidence. CDDRL Working papers, $n$. 42. Stanford Institute on International Studies, 2005.

CONASS - Conselho Nacional de Secretários da Saúde. O financiamento da saúde. Coleção para entender a gestão do SUS. v. 2, Brasília: CONASS, 2011. $124 \mathrm{p}$.

CONASS - Conselho Nacional de Secretários da Saúde. SUS: avanços e desafios. $1^{a}$ ed. Brasília: CONASS, 2006. 164 p.

DATASUS - Departamento de Informática do SUS. Indicadores de saúde. Disponível em: http://www2.datasus.gov.br/DATASUS/index.php?area=0201. Acesso em: 01 jul. 2015.

DIAS, J.; RUSSO, L. X. The Health Influence on Returns to Education in Brazil: a nonlinear approach. In: $42^{\circ}$ Encontro Nacional de Economia ANPEC, Natal, 2014.

DUARTE, A. J. M. et al. Transferências fiscais intergovernamentais no Brasil: uma avaliação das transferências federais, com ênfase no sistema único de saúde. Texto para discussão IPEA, n. 1451, 2009.

GERDTHAM, U-G.; JONSSON, B. International comparisons of health expedinture: theory, data and econometric analysis. In: CULYER, A. J.; NEWHOUSE, J. P. (Eds.). Handbook of Health Economics, v. 1A. Amsterdam: Elsevier, 2000.

GROSSMAN, M. On the concept of health capital and the demand for health. Journal of Political Economy, v. 80, p. 223-255, 1972.

GROSSMAN, M. The human capital model. In: CULYER, A. J.; NEWHOUSE, J.P. (Eds.) Handbook of Health Economics, v. 1A, Amsterdam: Elsevier, 2000. 
HAIR JR., J. F. et. al. Análise multivariada de dados. Porto Alegre: Bookman, 2005.

HOTELLING, H. Relations between two sets of variables. Biometrika, v. 28, n. 3/4, p. 321-377, 1936.

JOHNSON, R. A.; WICHERN, D. W. Applied multivariate statistical analysis. Englewood Clifs: Prentice-Hall, 1988.

KAISER, H. F. The varimax criterion for analytic rotation in factor analysis. Psychometrika, v. 23, n. 3, p. 187-200, 1958.

KICKBUSCH, I. Health promotion: not a tree but a rhizome. In: O'NEILL, M. et al. (Eds.). Health promotion in Canada: critical perspectives. Toronto: Canadian Scholars Press Inc. 2007.

MACKENBACH, J. P.; MEERDING, W. J.; KUNST, A. E. Economic implications of socio-economic inequalities in health in the European Union. Luxembourg: European Commission, 2007.

MARQUES, R. M.; MENDES, A. Os dilemas do financiamento do SUS no interior da seguridade social. Economia e Sociedade, v. 14, n. 1 (24), p. 159-175, jan./jun. 2005.

MÉDICI, A. C. Aspectos teóricos e conceituais do financiamento das políticas de saúde. In: PIOLA, S. F.; VIANNA, S. M. (Orgs.). Economia da saúde: conceitos e contribuição para a gestão da saúde. Brasília: IPEA, 1995.

MELAMED, C. Regulamentação, produção de serviços e financiamento federal do Sistema Único de Saúde: dos anos 90 aos 2000. In: MELAMED, C.; PIOLA, S. F. (Orgs.). Políticas públicas e financiamento federal do Sistema Único de Saúde. Brasília: IPEA, 2011.

MINCER, J. Schooling, earnings, and experience. New York: Columbia University Press, 1974.

OCDE - Organization for Economic Co-operation and Development. Health at a glance 2013. OCDE Indicators, 2013.

OCDE - Organization for Economic Co-operation and Development. Value for money in health spending. OECD Health Policy Studies, 2010.

OCKÉ-REIS, C. O. A constituição de um modelo de atenção à saúde universal: uma promessa não cumprida pelo SUS? Texto para discussão IPEA, n. 1376, fev./2009. 
PESQUISA revela que saúde pública é a principal preocupação dos eleitores. Disponível em: <http://g1.globo.com/jornal-nacional/noticia/2014/08/pesquisarevela-que-saude-publica-e-principal-preocupacao-dos-eleitores.html $>$ Acesso em: 04 ago. 2014.

PIOLA, S. F. et al. Financiamento público da saúde: uma história à procura de rumo. Texto para discussão IPEA, n. 1846, 2013.

PIOLA, S. F. et al. Saúde no Brasil: algumas questões sobre o Sistema Único de Saúde (SUS). Texto para discussão IPEA, n. 1391, 2009.

SERVO, L. M. S. et. al.; Financiamento e gasto público de saúde: histórico e tendências. In: MELAMED, C.; PIOLA, S. F. (Orgs.). Políticas públicas e financiamento federal do Sistema Único de Saúde. Brasília: IPEA, 2011.

VEGA, J.; IRWIN, A. Tackling health inequalities: new approaches in public policy. Bulletin of the World Health Organization, v. 82, n. 7, p. 482-483, 2004.

WEIL, D. N. Health and economic growth. IN: AGHION, P.; DURLAUF, S. N. (Eds.) Handbook of economic growth. North Holland, 2014.

WHO - World Health Organization. Closing the gap in a generation: health equity through action on the social determinants of health. Final Report of the Commission on Social Determinants of Health (CSDH). Geneva, 2008.

WILKINSON, R.; MARMOT, M. Social determinants of health: the solid facts. World Health Organization, 2003.

XU, K. et al. The determinants of health expenditure: a country-level panel data analysis. Working paper, World Health Organization, 2011. 


\section{Apêndice}

Tabela 7 - Indicadores sintéticos de saúde

\begin{tabular}{|l|c|c|c|c|c|c|}
\hline \multirow{2}{*}{ UFs } & \multicolumn{7}{c|}{ Indicadores Sintéticos de Saúde } \\
\hline & Demográfico & Socioeconômico & Mortalidade & Morbidade & Recursos & Cobertura \\
\hline Acre & 0.8409 & -0.6883 & 0.6861 & 0.4196 & 0.2828 & 0.2679 \\
\hline Alagoas & -0.4195 & -0.3950 & 0.2952 & 0.4359 & -0.4013 & 0.3256 \\
\hline Amazonas & 0.7820 & -0.5593 & 0.1784 & 0.5766 & 0.0022 & -0.3147 \\
\hline Amapá & 1.0316 & -0.5132 & 0.0540 & 0.0903 & -0.0515 & 0.5498 \\
\hline Bahia & -0.3031 & -0.3238 & 0.3618 & -0.1155 & -0.2364 & 0.1066 \\
\hline Cearán & -0.4947 & -0.5789 & -0.1572 & 0.1540 & -0.5430 & 0.2632 \\
\hline Distrito Federal & 1.3902 & -0.2951 & -0.4268 & -0.1588 & 1.6659 & -0.8400 \\
\hline Espírito Santo & 0.2329 & 0.5263 & -0.7605 & -0.3024 & 0.1340 & -0.0573 \\
\hline Goiás & 0.1305 & 0.6881 & -0.4332 & -0.4344 & -0.1438 & 0.2565 \\
\hline Maranhão & -0.1269 & -0.6481 & 0.6843 & 0.2946 & -0.6980 & -0.3427 \\
\hline Minas Gerais & -0.4542 & 0.6503 & -0.0329 & -0.3982 & 0.1248 & -0.0909 \\
\hline Mato Grosso Sul & 0.4095 & 0.5429 & -0.2731 & -0.3493 & -0.0922 & 0.4946 \\
\hline Mato Grosso & 0.6831 & 0.4629 & -0.3715 & -0.0601 & -0.0936 & 0.3731 \\
\hline Pará & 0.4517 & -0.2598 & 0.0950 & -0.0485 & -0.5401 & 0.0209 \\
\hline Paraíba & -1.0786 & -0.2899 & 0.5186 & 0.1489 & -0.2006 & -0.0822 \\
\hline Pernambuco & -0.9034 & -0.4922 & 0.1672 & 0.1453 & -0.3691 & 0.2982 \\
\hline Piauín & -0.8408 & -0.4956 & 0.4582 & -0.0794 & -0.2899 & -0.2131 \\
\hline Paranán & -0.2253 & 0.8133 & -0.4687 & -0.8263 & 0.0496 & 0.1187 \\
\hline Rio de Janeiro & -0.9397 & -0.0838 & -0.1419 & 0.3825 & 0.7009 & -0.5540 \\
\hline Rio Grande Norte & -0.4349 & -0.2521 & 0.5824 & 0.0629 & -0.3206 & -0.2406 \\
\hline Rondônia & 0.3718 & 0.6942 & -0.3479 & 0.0740 & 0.2193 & 0.6412 \\
\hline Roraima & 0.7367 & -0.5750 & 0.3455 & -0.0363 & 0.2498 & -0.9342 \\
\hline Rio Grande Sul & -0.8007 & 1.1598 & -0.7459 & -0.7511 & 0.1963 & -0.3762 \\
\hline Santa Catarina & 0.3274 & 1.3781 & -0.5597 & -0.1618 & 0.1786 & 0.0759 \\
\hline Sergipe & 0.0681 & -0.4603 & 0.5189 & 0.7515 & -0.5058 & 0.2279 \\
\hline São Paulo & -0.4510 & 0.2345 & -0.4199 & 0.6184 & 0.3565 & -0.1740 \\
\hline Tocantins & 0.0165 & -0.2401 & 0.1937 & -0.4322 & 0.3249 & 0.1996 \\
\hline Fonte: dado & & & & & \\
\hline
\end{tabular}

Fonte: dados da pesquisa. 
Tabela 8 - Indicadores fiscais

\begin{tabular}{|c|c|c|c|c|c|c|}
\hline \multirow[b]{2}{*}{ UFs } & \multicolumn{6}{|c|}{ Indicadores fiscas } \\
\hline & $\begin{array}{c}\text { Assistência } \\
\text { Social }\end{array}$ & Saúde & Educação & Saneamento & $\begin{array}{c}\text { Gestão } \\
\text { Ambiental }\end{array}$ & $\begin{array}{c}\text { Esporte e } \\
\text { Lazer }\end{array}$ \\
\hline Acre & 0.0128 & 0.0502 & 0.0759 & 0.0071 & 0.0030 & 0.0011 \\
\hline Alagoas & 0.0178 & 0.0235 & 0.0298 & 0.0053 & 0.0014 & 0.0001 \\
\hline Amazonas & 0.0071 & 0.0277 & 0.0242 & 0.0028 & 0.0007 & 0.0007 \\
\hline Amapá & 0.0107 & 0.0431 & 0.0727 & 0.0009 & 0.0007 & 0.0005 \\
\hline Bahia & 0.0178 & 0.0216 & 0.0213 & 0.0019 & 0.0009 & 0.0002 \\
\hline Ceará & 0.0285 & 0.0169 & 0.0376 & 0.0038 & 0.0030 & 0.0003 \\
\hline Distrito Federal & 0.0112 & 0.0123 & 0.0142 & 0.0008 & 0.0004 & 0.0004 \\
\hline Espírito Santo & 0.0196 & 0.0132 & 0.0144 & 0.0008 & 0.0002 & 0.0003 \\
\hline Goiás & 0.0174 & 0.0155 & 0.0217 & 0.0000 & 0.0003 & 0.0002 \\
\hline Maranhão & 0.0239 & 0.0152 & 0.0319 & 0.0049 & 0.0002 & 0.0004 \\
\hline Minas Gerais & 0.0136 & 0.0106 & 0.0163 & 0.0001 & 0.0007 & 0.0002 \\
\hline Mato Grosso Sul & 0.0235 & 0.0137 & 0.0214 & 0.0000 & 0.0005 & 0.0004 \\
\hline Mato Grosso & 0.0160 & 0.0139 & 0.0196 & 0.0001 & 0.0010 & 0.0004 \\
\hline Pará & 0.0181 & 0.0170 & 0.0194 & 0.0020 & 0.0003 & 0.0003 \\
\hline Paraíba & 0.0270 & 0.0234 & 0.0318 & 0.0015 & 0.0009 & 0.0001 \\
\hline Pernambuco & 0.0252 & 0.0240 & 0.0185 & 0.0017 & 0.0006 & 0.0001 \\
\hline Piauí & 0.0253 & 0.0297 & 0.0379 & 0.0006 & 0.0012 & 0.0005 \\
\hline Paraná & 0.0129 & 0.0101 & 0.0212 & 0.0002 & 0.0006 & 0.0001 \\
\hline Rio de Janeiro & 0.0114 & 0.0081 & 0.0166 & 0.0048 & 0.0009 & 0.0003 \\
\hline Rio Grande Norte & 0.0306 & 0.0265 & 0.0310 & 0.0002 & 0.0023 & 0.0002 \\
\hline Rondônia & 0.0073 & 0.0216 & 0.0313 & 0.0002 & 0.0006 & 0.0001 \\
\hline Roraima & 0.0116 & 0.0380 & 0.0685 & 0.0056 & 0.0002 & 0.0009 \\
\hline Rio Grande Sul & 0.0202 & 0.0090 & 0.0155 & 0.0001 & 0.0003 & 0.0000 \\
\hline Santa Catarina & 0.0089 & 0.0097 & 0.0142 & 0.0000 & 0.0002 & 0.0003 \\
\hline Sergipe & 0.0266 & 0.0244 & 0.0306 & 0.0043 & 0.0016 & 0.0003 \\
\hline São Paulo & 0.0082 & 0.0108 & 0.0203 & 0.0002 & 0.0009 & 0.0001 \\
\hline Tocantins & 0.0108 & 0.0342 & 0.0448 & 0.0010 & 0.0009 & 0.0008 \\
\hline
\end{tabular}

Fonte: dados da pesquisa. 
Tabela 9 - Correlação entre as variáveis originais (X1) e as variáveis canônicas - cargas canônicas

\begin{tabular}{|c|c|c|c|c|c|c|c|c|}
\hline \multirow{3}{*}{ VañavelX1 } & \multirow{2}{*}{\multicolumn{2}{|c|}{ 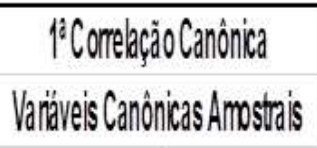 }} & \multirow{2}{*}{\multicolumn{2}{|c|}{ 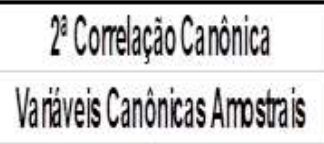 }} & \multirow{2}{*}{\multicolumn{2}{|c|}{$\begin{array}{l}3^{3} \text { Comelaç̧ào Canônica } \\
\text { Vanäuveis Canônicas Amostais }\end{array}$}} & \multirow{2}{*}{\multicolumn{2}{|c|}{ 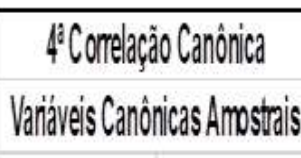 }} \\
\hline & & & & & & & & \\
\hline & $U 1$ & VI & U1 & VI & $\| 1$ & V & U1 & VI \\
\hline Denmografia & 0.1708 & -0.3365 & 1.9813 & 0.0298 & -2.9787 & 0.0184 & -1.5960 & 0.1494 \\
\hline Socioeconômico & 1.3057 & 12160 & -1.8304 & .308090 & 0.3267 & 3.5714 & 1.7504 & $25: 487$ \\
\hline Mortalidade & -0.7542 & -0.9798 & .1 .6660 & 1.0962 & 21182 & .2 .4202 & 3.1958 & -20814 \\
\hline Morbidade & 0.3913 & -0.1620 & 0.0307 & 1.5881 & -8.1323 & .1 .3750 & .60420 & -1.77788 \\
\hline Recursos & -0.7630 & .08791 & -1.90907 & 0.4042 & 2.4792 & .06090 & 1.5600 & -0.0650 \\
\hline Cobertura & -3.3505 & .0 .3319 & .16484 & .1 .2075 & 2.6763 & 1.0818 & 2.0527 & 12.2698 \\
\hline
\end{tabular}

Fonte: dados da pesquisa.

Tabela 10 - Correlação entre as variáveis originais (X2) e as variáveis canônicas - cargas canônicas

\begin{tabular}{|c|c|c|c|c|c|c|c|c|}
\hline \multirow{3}{*}{ VariávelX2 } & \multicolumn{2}{|c|}{ 1a Correlação Canônica } & \multicolumn{2}{|c|}{ 2a Correlação Canônica } & \multicolumn{2}{|c|}{ 3a Correlação Canônica } & \multicolumn{2}{|c|}{ 4a Correlação Canônica } \\
\hline & \multicolumn{4}{|c|}{ Variáveis Canononicas Amostrais Variáaveis Canônicas Amostra } & VariáveisCa & cas Amostrais & Variáveis Car & las Amostrais \\
\hline & U1 & V1 & U1 & V1 & U1 & V1 & U1 & V1 \\
\hline Assistência Social e Previd. & 1.4507 & 0.4836 & 2.1005 & .0 .2637 & 1.9054 & .0 .2131 & 0.4605 & 0.0395 \\
\hline Saide & 1.6427 & 0.2354 & .0 .0758 & 0.6943 & .0 .0737 & 0.6050 & .0 .2083 & 0.8605 \\
\hline Saneamento & .0 .8485 & .0 .3340 & .0 .4740 & .0 .7099 & .0 .4624 & .0 .6310 & .0 .4132 & -0.6185 \\
\hline Educaçãoe Cultura & 0.9433 & 0.0591 & 0.3300 & -0.3527 & 0.2622 & .0 .3000 & 0.0041 & 0.5324 \\
\hline Gestão Ambiental & -1.7565 & 0.5985 & .1 .3514 & 0.2538 & -1.2382 & 0.2261 & .0 .2006 & 0.1505 \\
\hline Esporte e Lazer & .0 .8601 & .0 .0839 & .1 .8363 & .02023 & -1.6378 & 0.1586 & 0.3780 & 0.1970 \\
\hline
\end{tabular}

Fonte: dados da pesquisa. 